\title{
Inferior alveolar nerve paresthesia caused by periapical infection: A case report
}

\author{
Inês Ferreira ${ }^{1}$, Paula Rodrigues ${ }^{2}$ and Irene Pina-Vaz ${ }^{1}$ \\ ${ }^{1}$ Faculty of Dental Medicine of University of Porto, Porto, Portugal \\ ${ }^{2} \mathrm{DMD}$, Allmed Clinic, Braga, Portugal
}

\begin{abstract}
The aim of this paper was to describe a case of inferior alveolar nerve infection-induced paresthesia and to discuss the most appropriate diagnostic tools. Although radiographically, the periapical lesion didn't appear to be in close vicinity to the mandibular nerve canal, the persistence of the symptomatology led to the suspicion of an inferior alveolar nerve compression. A cone-beam computed tomography (CBCT) scan showed an impressive lesion with great bone destruction, in direct contact with the inferior alveolar nerve, compressing it. Besides a complete medical history, a CBCT scan may be crucial to identify the cause of the paresthesia, supporting the clinical exam and preventing an irreversible nerve injury.
\end{abstract}

\section{Introduction}

Paresthesia consists of a sensation of numbness or tingling, resulting from neural injury [1]. About half of the cases concerning facial paresthesia originate in procedures or dental pathology, with the inferior alveolar nerve (IAN) and the mental one being most frequently affected [2]. It can be attributed to a variety of systemic (microbial infections, multiple sclerosis, lymphoma or diabetes mellitus) and/or local etiological factors, such as mandibular fractures, cysts, impacted teeth, infections (osteomyelitis, apical periodontitis, peri-implantitis), and iatrogenic lesions $[2,3]$.

Diagnosis of paresthesia requires an accurate anamnesis, determining the onset of the sensory alteration and its evolution. Examination of the affected area can be carried out using thermal, mechanical or electrical tests that elicit subjective responses [2]. Periapical and panoramic radiographs must be carried out to determine the cause of paresthesia. A cone-beam computed tomography (СBCT) scan will be helpful in displaying small anatomical structures and revealing their spatial relations $[2,4]$.

Endodontic-related paresthesia is a rare complication in endodontics and can be related to periapical pathology (infection) or endodontic iatrogenic causes, as a consequence of incorrect treatment of the root canals (over-instrumentation or overfilling), resulting in mechanical pressure and/or chemical injury [2]. In the first situation most of the cases have been reported in lower pre-molars or molars, with large periradicular lesions $[1,4-6]$. The symptoms generally subside trough elimination of infection by root canal treatment or immediate extraction of the affected tooth. Nevertheless, if the condition is severe, an irreversible neural lesion may result from it [2].

The aim of this report is to describe a case of IAN paresthesia due to the expanding endodontic infection, not clearly detected by the conventional radiographic exam, and the use of CBCT, with enhanced sensitivity, to aid the diagnostic, supporting the clinical exam, and thus influencing the treatment plan.

\section{Case report}

In June 2018 a 45 -year-old woman was referred for endodontic retreatment of the lower right second molar (\#47). The patient reported a tingling sensation and numbness, of sudden appearance, on the right side of the face, after finishing the antibiotics therapy for an acute apical abscess, a week ago. The symptoms for abscess disappeared, but a numbness of the lower lip started after that. The tooth had been endodontically treated five years before and had already presented some acute episodes, which subsided after antimicrobial prescription. It presented a composite restoration. Clinically, the palpation and percussion were normal. Paresthesia was detected in the right half of the lower lip, developing over a week. The radiographic exam (panoramic radiograph) revealed an apical radiolucency associated with the tooth \#47 (Figure 1). In the medical history it was reported a recent cerebrovascular accident (CVA) with symptoms (numbness in the lower lip) that were identified as similar to those of the current dental paresthesia. Although, radiographically, the periapical lesion didn't appear to be in close vicinity to the mandibular nerve canal, the persistence of the symptomatology led to the suspicion of an inferior alveolar nerve compression. The patient was concerned about the prognosis and the potential risk of irreversible damage to the alveolar nerve, therefore consenting to further radiographic imaging. The neurologic examination was negative, except for the hypoesthesia of the lip, excluding other medical causes. A CBCT scan was undertaken, clearly showing an impressive lesion with great bone destruction, in direct contact with the inferior alveolar nerve, compressing it (Figures 2 and 3).

*Correspondence to: Irene Pina-Vaz, Faculty of Dental Medicine of University of Porto, Porto, Portugal, E-mail: igapv@sapo.pt

Key words: apical periodontitis, cone beam computed tomography, inferior alveolar nerve, paresthesia

Received: November 09, 2018; Accepted: November 23, 2018; Published: November 27, 2018 


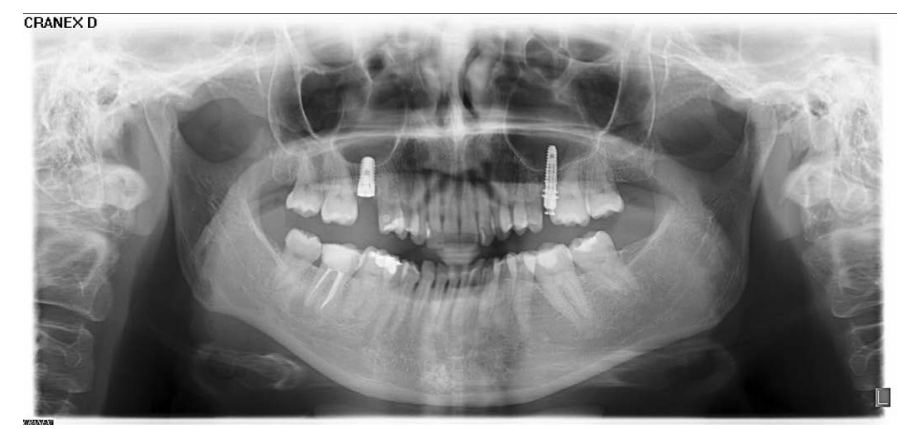

Figure 1. Panoramic radiograph showing a radiolucent area in the apical region of the second lower right molar (\#47)

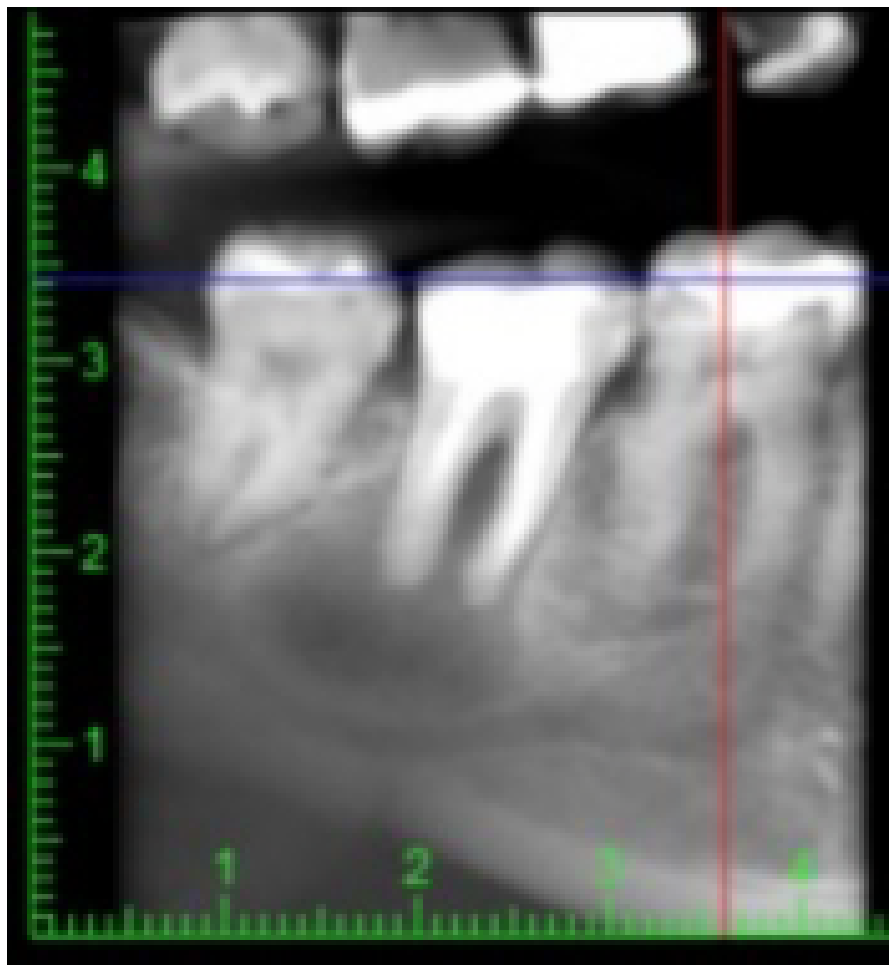

Figure 2. Reconstruction (CBCT) showing the compression of the inferior alveolar nerve, in the proximity of the apical region of tooth \# 47

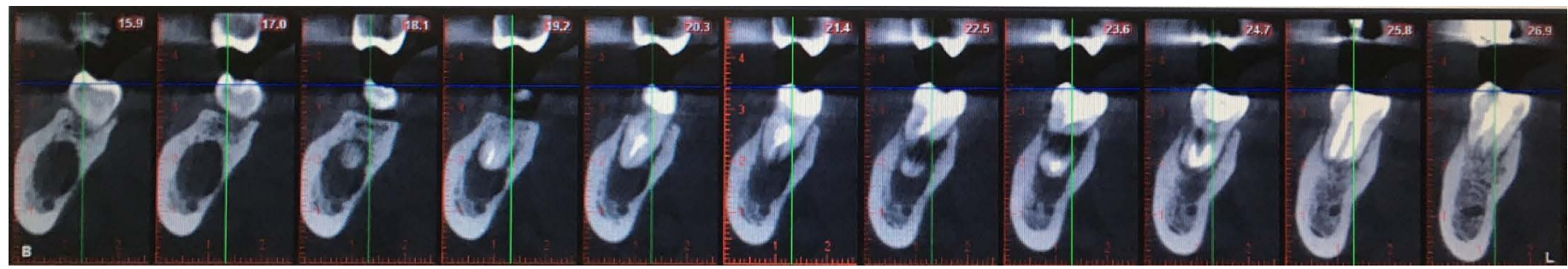

Figure 3. CBCT examination (coronal slices/cuts) of tooth $\# 47$ revealing the relationship between the periapical lesion and the mandibular canal

After discussing treatment options with the patient, it was decided to extract the tooth with surgical removal of the lesion, in order to rapidly remove the mechanical compression, preventing permanent nerve damage. Besides, CBCT images excluded a more conservative approach. Although the patient agreed with the surgery, after nearly 2 weeks, as the symptoms subsided, she missed the appointment and the attempts to call her again were unsuccessful.

\section{Discussion}

Apical periodontitis in root-canal treated teeth is often associated to endodontic therapy of low quality $[7,8]$. In the present case, iatrogenic errors, such as overfilling or material extrusion, were not detected. On the contrary, the CBCT showed a root canal filling densely compacted, associated with an extensive apical bone destruction. 
Paresthesia induced by periapical infection is described as a result of mechanical pressure on the nerve fibers, due to the expanding infectious process, or even by the damage microbial products can infer in the nerve conduction [1]. In the present report, the great extension of the lesion, only appreciated after CBCT scan, associated with the clear compression of the IAN, could explain the persistent numbness presented by the patient. Through CBCT images it was perceptible that the bone barrier between the apex of the affected tooth, already weakened by the presence of perirradicular infection, caused the mandible canal to become more vulnerable to the mechanical and microbial aggression from the root canal to the nerve endings. The risk of a permanent nerve injury [9] and the patient's concern led to a more aggressive approach, aiming to remove the cause as early as possible, in order to reach a better prognosis [5]. Most of the dental paresthesia cases have implicated lower PMs [1] and a few lower or superior molars $[2,4,6]$. In case of the 1 st mand molar, the distance between the apices and the mandibular canal varies between 1-4 mm, being inferior to 1 $\mathrm{mm}$ with the 2 nd and $3 \mathrm{rd} \mathrm{M} \mathrm{[6].} \mathrm{In} \mathrm{addition,} \mathrm{in} \mathrm{the} \mathrm{posterior} \mathrm{region,}$ the mandibular bone is not very dense [10].

Once the cause is determined and removed, paresthesia is usually resolved within days $[5,10]$. The chosen treatment depends on the cause and extent of injury [2].

Regardless of the affected tooth, it is emphasized the importance of an accurate anamnesis, evaluating the beginning of the sensorial alteration and its evolution. In the case here presented, this aspect was crucial in the decision of requiring auxiliary radiographic exams, which led to an accurate diagnosis, preventing other treatments the patient could not benefit from. CBCT images revealed relevant information about the neighbouring structures to the IAN canal and periapical lesion to the cortical plates, with higher sensitivity than conventional radiography, [11]. In the present situation, not only did CBCT support the clinical exam but, more importantly, it highlighted the severity of the lesion, advising against a more conservative approach. Furthermore, $\mathrm{CBCT}$ can also be an important additional resource in the prevention of paresthesia of endodontic origin [2]. The early detection of bone lesions that can be in intimate anatomic relationship with dental apexes and neural structures enables either a preventable procedure with a cautious treatment plan or even an immediate treatment before irreversible damage occurs.

\section{Conclusion}

Diagnosis of endodontic-related inferior alveolar nerve paresthesia is based on a complete medical history, assessing the onset of symptoms. A CBCT scan may be crucial to identify the cause or support the clinical diagnosis, that way preventing an irreversible nerve injury.

\section{References}

1. Di Lenarda R, Cadenaro M, Stacchi C (2000) Paresthesia of the mental nerve induced by periapical infection: a case report. Oral Surg Oral Med Oral Pathol Oral Radiol Endod 90: 746-749. [Crossref]

2. Alves FR, Coutinho MS, Goncalves LS (2014) Endodontic-related facial paresthesia systematic review. J Can Dent Assoc 80: e13. [Crossref]

3. Pogrel MA, Thamby S (1999) The etiology of altered sensation in the inferior alveolar lingual, and mental nerves as a result of dental treatment. $J$ Calif Dent Assoc 27: 534538. [Crossref]

4. Gambarini G, Plotino G, Grande NM, Testarelli L, Prencipe M, et al. (2011) Differential diagnosis of endodontic-related inferior alveolar nerve paraesthesia with cone beam computed tomography: a case report. Int Endod J 44:176-181. [Crossref]

5. Censi R, Vavassori V, Borgonovo AE, Re D (2016) Infection Related Inferior Alveolar Nerve Paresthesia in the Lower Premolar Teeth. Case Rep Dent 2016: 2623507. [Crossref]

6. Gonzalez-Martin M, Torres-Lagares D, Gutierrez-Perez JL, Segura-Egea JJ (2010) Inferior alveolar nerve paresthesia after overfilling of endodontic sealer into the mandibular canal. $J$ Endod 36:1419-1421. [Crossref]

7. Melo L, Ferreira I, Lacet G, Braga AC, Pina-Vaz I (2017) Apical periodontitis and ora status in patients with different systemic conditions and smoking habits in Portuguese population. Revista Endodoncia 35:10-22.

8. Tavares PB, Bonte E, Boukpessi T, Siqueira JF Jr, Lasfargues JJ (2009) Prevalence of apical periodontitis in root canal-treated teeth from an urban French population: influence of the quality of root canal fillings and coronal restorations. J Endod 35:810813. [Crossref]

9. Pogrel MA (2007) Damage to the inferior alveolar nerve as the result of root canal therapy. J Am Dent Assoc 138: 65-69. [Crossref]

10. Tilotta-Yasukawa F, Millot S, El Haddioui A, Bravetti P, Gaudy JF (2006) Labiomandibular paresthesia caused by endodontic treatment: an anatomic and clinical study. Oral Surg Oral Med Oral Pathol Oral Radiol Endod 102: e47-59. [Crossref]

11. Estrela C, Bueno MR, Leles CR, Azevedo B, Azevedo JR (2008) Accuracy of cone beam computed tomography and panoramic and periapical radiography for detection of apical periodontitis. J Endod 34:273-279. [Crossref]

Copyright: (C2018 Ferreira I. This is an open-access article distributed under the terms of the Creative Commons Attribution License, which permits unrestricted use, distribution, and reproduction in any medium, provided the original author and source are credited. 